Commentary

Invited Commentary

Central Eur J Paed 2020;16(2):202-203

DOI $10.5457 / \mathrm{p} 2005-114.278$

\title{
Comprehensive Approach to Vulnerable Adolescents with Mental Health Problems
}

Vida Jeremic Stojkovic

Department of Humanities, Faculty of Medicine, University of Belgrade, Pasterova 2, 11000 Belgrade

Correspondence: vidajeremic@yahoo.com; Tel.: + 38160636 1076; Fax.: 381113636300

Received: June 16, 2020; Accepted: June xx, 2020

Key Words: Adolescents • Mental Health - Social Aeterminants of Health.

Estimates suggest that about $20 \%$ of children and adolescents across the globe have mental health challenges (1). The social determinants of health are paramount for youth living with mental health difficulties (2). The social and economic factors influence mental health outcomes as well as the quality of healthcare among youth (2). Previous evidence showed that the behavioral problems are more common among adolescents living in poverty (3). In fact, inadequate housing, substandard education, and disruptive collective socialization of adolescents result from chronic poverty (3). A systematic review of studies exploring the association between socioeconomic inequalities and mental health in children and adolescents suggested that the risk of developing mental health disorders among socio-economically disadvantaged children and adolescents is increased two to three-fold compared to more affluent children and adolescents (4). These findings highlight the fact that in order to achieve optimal health outcomes in a population of vulnerable adolescents, social determinants of health must be incorporated in the management of mental health problems.

In this issue Goodday presents an approach for vulnerable adolescents with mental health difficulties, which revolves around the hypothetical case scenario (5). The case presents an adolescent who had psychotic symptoms, who abused cannabis and tobacco, and was admitted to a mental health inpatient unit. The patient responded well to the treatment, but the outpatient care was unsatisfying due to his underprivileged socio-economic background (i.e. the patient did not have a permanent place of residence, was not able to continue his education and wasn't able to take the prescribed therapy regularly). By focusing on the ethical issues surrounding this case, specific gaps throughout his transition from mental health inpatient unit to the community are being addressed. Finally, it is concluded that the social structure was not supportive enough for this adolescent to function optimally as well as to adhere to healthcare recommendations (such as to regularly attend counseling appointments and to follow his treatment).

Empirical data suggest that mental health services are often inflexible, poorly coordinated and do not address vulnerable adolescents' needs (6). Adolescents with poor psychosocial adjustment, who belong to racial and ethnic minorities, who were poor and whose parents had lower educational attainment were more strongly affected by the shortcomings in the health care system. Indeed, inflexibility of services has previously been identified as one of the main barriers for youth from at-risk groups including ethnic and sexual minorities, culturally and linguistically diverse, homeless, 
substance users and youth residing in remote and rural areas to access mental health services (7).

Goodday's harm reduction approach (5) adequately incorporates to findings from the studies about barriers to accessing mental health services reported by homeless youth, such as poor motivation for treatment, negative past experiences with services, limited service options, costs, waiting lists, treatment availability (7). According to these accounts, the main facilitators to access mental health services among homeless youth were therapeutic alliance and diversity of treatment options (7).

Further evidence showed that a minority of adolescents of low socio-economic status actually use mental health services (8). Furthermore, it has been previously observed that mental health programs that did not take into account the social and economic context of family life, living conditions, inadequate housing and lack of employment have limited effectiveness in real-life circumstances (3). A cross-national study of the association between social support and mental health among vulnerable adolescents living in economically deprived areas showed that the improvement of social support in families and neighborhoods may lessen the distress among these individuals (9). Therefore, harm reduction approach, which takes into account the social context of vulnerable patients, must incorporate specific needs of disadvantaged youth to achieve optimum outcomes.

In conclusion, there is a need for a more meaningful involvement of the health care system to help the vulnerable youth overcome barriers in seeking mental healthcare services.

Conflict of Interest: The author declares that she has no conflict of interest.

\section{References}

1. World Health Organization. 10 Facts on mental health [Cited 2020 June 10]. Available at: http://www.who.int/ features/factfiles/mental_health/mental_health_facts/en.

2. Patel V, Flisher AJ, Hetrick S, McGorry P. Mental health of young people: a global public-health challenge. Lancet. 2007;369(9569):1302-1313. doi:10.1016/S01406736(07)60368-7

3. Dashiff C, DiMicco W, Myers B, Sheppard K. Poverty and adolescent mental health. J Child Adolesc Psychiatr Nurs. 2009;22(1):23-32. doi:10.1111/j.17446171.2008.00166.x

4. Reiss F. Socioeconomic inequalities and mental health problems in children and adolescents: a systematic review. Social science \& medicine. 2013;90:24-31.

5. Goodday M. Considering Social Determinants of Health: A Case for the Just Treatment of Vulnerable Adolescent Mental Health Inpatients. Central European Journal of Paediatrics 2020;16(2):161-67.

6. Witt WP, Kasper JD, Riley AW. Mental health services use among school-aged children with disabilities: The role of sociodemographics, functional limitations, family burdens, and care coordination. Health Services Research. 2003;38:1441-66.

7. Brown A, Rice SM, Rickwood DJ, Parker AG. Systematic review of barriers and facilitators to accessing and engaging with mental health care among at-risk young people. AsiaPacific Psychiatry. 2016;(1):3-22.

8. Cauce AM, Domenech-Rodríguez M, Paradise M, Cochran BN, Shea JM, Srebnik D, et al. Cultural and contextual influences in mental health help seeking: a focus on ethnic minority youth. Journal of consulting and clinical psychology. 2002;70(1):44.

9. Cheng Y, Li X, Lou C, Sonenstein FL, Kalamar A, Jejeebhoy $S$, Delany-Moretlwe $S$, et al. The association between social support and mental health among vulnerable adolescents in five cities: findings from the study of the wellbeing of adolescents in vulnerable environments. Journal of Adolescent Health. 2014;55(6):S31-8. 